ORIGINAL RESEARCH

\title{
Cerebrovascular Dynamics and Vascular Endothelial Growth Factor in Acute Mountain Sickness
}

\author{
Joseph Palma, MD; Christian Macedonia, MD; Patricia Deuster, PhD; Cara Olsen, MPH; \\ B. Robert Mozayeni, MD; Kevin E. Crutchfield, MD
}

From the Departments of Military and Emergency Medicine (Drs Palma and Deuster), Biostatistics (Dr Olsen), Obstetrics and Gynecology (Dr Macedonia), and Neurology and Military Emergency Medicine (Dr Crutchfield), and the Human Performance Laboratory (Dr Deuster), Uniformed Services University of the Health Sciences, Bethesda, MD; and New Health Sciences Inc, Rockville, MD (Drs Mozayeni and Crutchfield).

Objective.-To determine if serum vascular endothelial growth factor (VEGF) and ultrasonic monitoring of vascular dynamics with dynamic vascular analysis at sea level and high altitude correlate with acute mountain sickness symptoms.

Methods.-Nine volunteers participated in a staged ascent from sea level to $4300 \mathrm{~m}$ undergoing complete transcranial Doppler studies with dynamic vascular analysis. Serum VEGF levels, Lake Louise scores, Spielberger-1 scores, Subjective Exercise Experiences Scale positive scores, and Symptom Checklist-90 surveys were collected after 24 hours at each altitude.

Results.-Symptom scores, index of pulsatility, and dynamic flow index differentiated the subjects into 2 distinct groups. Symptomatic subjects had increased VEGF levels at sea level but decreased levels at $4300 \mathrm{~m}$. The dynamic flow index increased in symptomatic subjects at $4300 \mathrm{~m}$ compared with the asymptomatic subjects. The mean flow velocity increased in both groups and could not be used to differentiate the subjects.

Conclusions.- Altered vascular physiology is associated with acute mountain sickness. Increased vascular permeability increases vascular capacitance, with an increase in dynamic flow index to meet these demands. Altered vascular dynamics were associated with high-altitude cerebral edema in 1 subject. Dynamic vascular analysis demonstrated altered vascular pathophysiology associated with acute mountain sickness. Changes in VEGF were meaningful when interpreted with the dynamic vascular analysis findings. These physiological findings may help explain the vascular changes associated with hypocarbic hypoxemia at altitude.

Key words: dynamic vascular analysis, transcranial Doppler ultrasound, acute mountain sickness, endothelial dysfunction, blood brain barrier, vascular endothelial growth factor, altitude

\section{Introduction}

Acute mountain sickness (AMS) represents an increasing clinical problem at higher altitude ${ }^{1,2}$; if unrecognized and untreated, it may lead to death in just a few hours. Although well documented, no conclusive physiological evidence exists to link increased cerebral blood flow (CBF) secondary to hypobaric hypoxia to the development of AMS or high-altitude cerebral edema. Serum vascular endothelial growth factor (VEGF) levels have been measured in subjects exposed to altitude; however,

Corresponding author: Kevin E. Crutchfield, MD, New Health Sciences Inc, 6903 Rockledge Dr, Suite 230, Bethesda, MD 20817-1818 (e-mail: kevin.crutchfield@newhealthsciences.com). changes do not correlate with development of altitude illness. $^{3-5}$ Hypoxemia constricts pulmonary vessels, ${ }^{6}$ whereas in the cerebrum it causes hyperemia. ${ }^{7}$ However, both vascular territories develop endothelial dysfunction with edema at higher altitude. Human transcranial Doppler (TCD) studies at high altitude revealed increased middle cerebral artery (MCA) mean flow velocities (MFVs) secondary to hypoxemic drive, ${ }^{8}$ yet changes in MFV in the MCA do not predict subjects who develop AMS. ${ }^{9}$ Transcranial Doppler studies of the MCA at altitude have demonstrated diminished index of pulsatility (PI) or index of resistance ${ }^{10-12}$ but with unclear clinical implications.

We investigated the impact of increasing altitude as a 
function of graded hypoxemic stress by correlating $\mathrm{CBF}$ dynamics informed by dynamic vascular analysis (DVA $)^{13}$ of TCD-derived velocimetry data, serum VEGF levels, and 4 sickness symptom scales. We utilized DVA to assess cerebrovascular physiology, allowing for sensitive monitoring of changes in cerebrovascular physiology associated with changes in altitude.

\section{Methods}

The institutional review board at the Uniformed Services University of the Health Sciences, Bethesda, MD, approved this prospective, longitudinal, single-arm, singleblind pilot trial. Nine healthy, athletic male subjects who were medical faculty and $\mathrm{PhD}$ candidates from the Uniformed Services University of the Health Sciences who signed informed consent forms were taken from sea level to the summit of Pikes Peak, CO, at 4300 m (altitude). The ascent up the mountain was staged for 5 days with daily stops and testing at $1585 \mathrm{~m}, 2500 \mathrm{~m}$, and $3415 \mathrm{~m}$ and a final ascent to $4300 \mathrm{~m}$ (summit). All volunteers were given the following questionnaires at each altitude to measure severity of altitude illness: Lake Louise score, Spielberger-1 score, Subjective Exercise Experiences Scale positive score, and Symptom Checklist-90 surveys. ${ }^{14-17}$ Transcranial Doppler data were acquired by a single technologist (nonsubject) from recumbent subjects at each altitude by well-documented handheld techniques and depth of measurement ${ }^{18}$ with a Pioneer Companion system (Nicolet Vascular, Golden, CO) having a 2-MHz probe. Velocimetry measurements were taken from both MCAs M1 (50-60 mm), both anterior cerebral arteries A1 $(60-70 \mathrm{~mm})$, both posterior cerebral arteries, both P1 $(60-70 \mathrm{~mm})$ and P2 $(60-70 \mathrm{~mm})$, both vertebral arteries $(60-65 \mathrm{~mm})$, and the basilar artery (80-100 mm). New Health Sciences Inc (Rockville, MD) performed the DVA analysis according to previously reported methods. ${ }^{13}$ This analysis now includes the dynamic flow index (DFI $=$ MFV/PI), dynamic pressure index (DPI $=$ natural $\log$ of systolic acceleration)/ PI), and dynamic compliance index (DCI = natural log of systolic acceleration/MFV). The DFI relates MFV to resistance (PI) and reflects small vessel effects on flow through conductance vessels. The DPI relates acceleration or force of flow to the downstream resistance and relates the effects of small vessel volume on the force of flow or the pressure gradient on which the blood flows. The DCI relates acceleration or force of flow to MFV and reflects the elasticity and kinetic efficiency of pulse wave propagation in the vessel segment insonated.

Blood samples were taken at each altitude and immediately centrifuged at $3400 \mathrm{~g}$ for 20 minutes to separate the serum, which was subsequently flash frozen in the field and sent for proteomic VEGF characterization by the Advanced Concepts Laboratory of the National Cancer Institute, National Institutes of Health, Bethesda, MD (Dr Steven Libuttis).

All 9 subjects completed the study up to $3415 \mathrm{~m}$. Only 8 subjects fully completed the study at summit altitude. One subject experienced acute altitude illness 2 hours after ascent, with ataxia, bilateral papilledema, confusion, and disorientation to person, place, and time. He recovered spontaneously without sequelae after emergent descent to $3650 \mathrm{~m}$. Transcranial Doppler velocimetry data were collected from this subject at the summit before descent, but no blood sample or symptom scores were obtained. Symptom scores were all assumed to be severe in this subject because he was incapable of answering the questionnaires at altitude. To include him in the analysis, he was assigned a total Lake Louise score of 30, a Symptom Checklist-90 score of 90, and a Spielberger-1 score of 60 at $4300 \mathrm{~m}$. Nonparametric statistical methods based on ranks were used for all analysis involving symptom scales, so the absolute numerical score was not as important because the scores were higher than those recorded by the other subjects.

Mean flow velocity, systolic upstroke acceleration (SA), PI, VEGF, and symptom scores were compared by using the Wilcoxon signed rank test. A significant difference was considered at $P<.05$. Symptom scores at altitude were compared with MFV, natural log of systolic acceleration, PI, VEGF, DFI, DPI, and DCI scores at all altitudes by using Spearman rank correlation (r). Spearman rank correlations lie between +1 and -1 , and a correlation of 0 indicates no relationship. A positive correlation indicates that an increase in that measure corresponds to an increase in symptoms, whereas a negative correlation indicates that a decrease in that measurement corresponds to a decrease in symptoms. The analysis focused on the relationship to symptom scales at final altitude because subjects experienced varied symptoms at lower altitudes.

Symptom scores were compared with changes in the other variables as follows:

- For each subject, the slope of the linear regression for the PI at each altitude was calculated. A positive slope indicated that PI increased with increasing altitude; conversely, a negative slope indicated that PI decreased with increasing altitude.

- Spearman rank correlation was used to compare the slope of the regression with a symptom score for every subject. A negative correlation indicated that a subject whose PI increased with increased altitude (positive slope) had fewer symptoms.

- This analysis was repeated for every variable. 


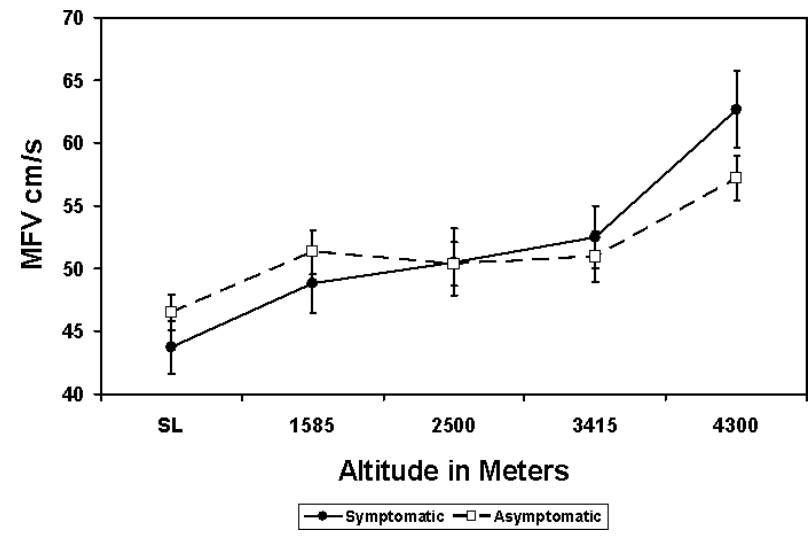

Figure 1. Effect of altitude on mean flow velocity (MFV). Both symptom groups showed increased MFV with altitude.

- For each TCD measurement, average change from sea level to $4300 \mathrm{~m}$ was calculated for symptomatic $(\mathrm{Sx})$ and asymptomatic (Asx) subjects, and the 2 groups were compared by using the paired-samples $t$ test.

\section{Results}

We observed changes in TCD values, confirming increased MFV with increasing altitude in every subject (Figure 1), with a holocephalic, median level increasing from $46.64 \mathrm{~cm} \cdot \mathrm{s}^{-1}$ at sea level to a median of 55.32 $\mathrm{cm} \cdot \mathrm{s}^{-1}$ at altitude $(P=.008)$. There was no significant cohort change in holocephalic PI with increasing altitude: the PI increased in 5 subjects and decreased in 4 subjects.

There was no cohort change in mean VEGF level with altitude: 5 subjects experienced increased VEGF, 2 subjects had no change, and 2 subjects had a peak value at $3415 \mathrm{~m}$ with a decrease in VEGF at summit altitude.

Symptoms scales increased for most subjects with increasing altitude. The median number of reported symptoms increased from 1 at sea level to 15 at altitude $(P$ $=.008)$ on the Symptom Checklist-90 scale, from 23 at sea level to 25 at altitude $(P=.063)$ on the Spielberger1 scale, and from 1 at sea level to 9 at altitude $(P=$ .008 ) on the Lake Louise questionnaire (Figure 2).

Subjects with a high PI at every altitude above sea level reported fewer symptoms at final altitude $(-0.04$ $<\rho<-0.79)$. Subjects whose PI increased with increasing altitude had far fewer symptoms than those subjects whose PI decreased with increasing altitude (Figure 3 ). Because of this relationship, the group was subcategorized into an Sx group with 3 subjects and an Asx group with 6 subjects (Table 1).

Comparisons of TCD, DVA, and VEGF levels between the 2 groups at sea level and altitude, as well as

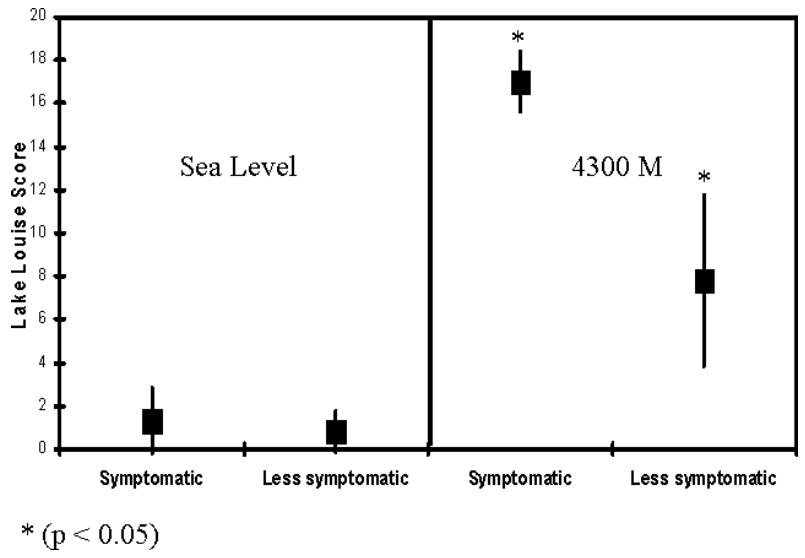

Figure 2. Effect of summit altitude $(4300 \mathrm{~m})$ on Lake Louise symptom scores. The difference in symptom scales between the symptomatic group $(n=3)$ and the asymptomatic group $(n=6)$ became significant at peak altitude. Means and ranges for Lake Louise scores are shown. All symptom scales showed similar differences.

analysis comparing changes between sea level and altitude within the Sx and the Asx groups, were performed (Table 2). At altitude, we noted significant group differences $(P<.05)$ between the groups regarding PI (Figure 3 ) and DFI (Figure 4). Trending, but not a significant difference, was noted in the DPI and the serum VEGF level (Figure 5). We noted a dramatic drop in serum VEGF level at high altitude in the Sx group. This drop indicates complex kinetics of serum VEGF in hypoxic environments.

\section{Discussion}

Noninvasive monitoring of cerebrovascular dynamics with changing altitude revealed that increased hypox-

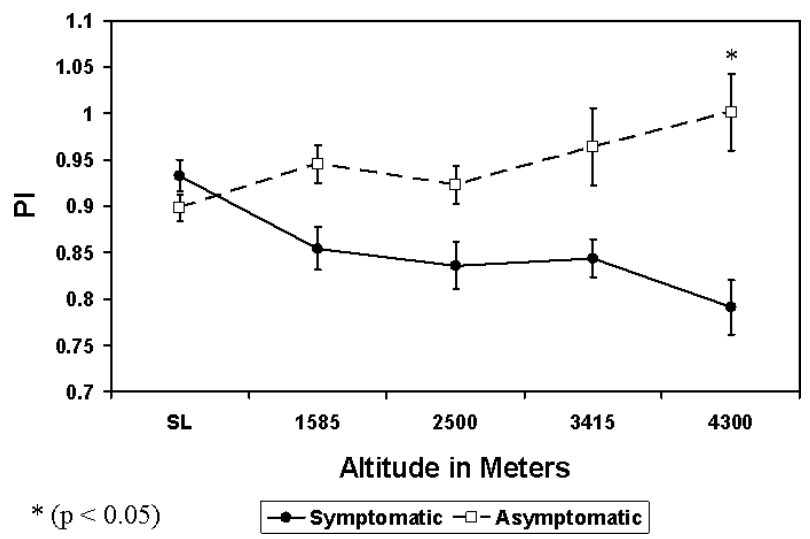

Figure 3. Effect of altitude on index of pulsatility (PI). Changes in PI significantly differed between the 2 symptom groups with altitude $(* P<.05$; paired-samples $t$ test $)$. 
Table 1. Ranges of symptom scales at sea level and altitude $(4300 \mathrm{~m})$ between the symptomatic (Sx) and asymptomatic (Asx) groups

\begin{tabular}{|c|c|c|c|c|}
\hline \multirow[b]{2}{*}{ Scale } & \multicolumn{2}{|c|}{ Sea level } & \multicolumn{2}{|c|}{ Altitude } \\
\hline & Mean & Range & Mean & Range \\
\hline Symptom Checklist-90 Sx & 5 & $-0.29-10.29$ & 53 & $37.44-68.56$ \\
\hline Symptom Checklist-90 Asx & 2.17 & $-2.66-7.00$ & 15.33 & $7.75-22.91$ \\
\hline Spielberger-1 Sx & 27.33 & $18.95-35.22$ & 46.00 & $38.91-53.07$ \\
\hline Spielberger-1 Asx & 23.33 & $20.53-26.14$ & 24.83 & $19.45-30.22$ \\
\hline Lake Louise total $\mathrm{Sx}$ & 1.33 & $-0.20-2.86$ & 17.00 & $15.59-18.41$ \\
\hline Lake Louise total Asx & 0.83 & $-0.15-1.82$ & 7.83 & $3.86-11.80$ \\
\hline Subjective Exercise Experiences Scale score Sx & -12.67 & $-18.78--6.56$ & 36.5 & $24.48-48.52$ \\
\hline Subjective Exercise Experiences Scale score Asx & -12.67 & $-19.76--5.58$ & 3.0 & $6.24-12.94$ \\
\hline
\end{tabular}

emia in the Sx group was associated with increasing DFI and diminished pulsatility, suggesting reduced vascular resistance from expanding capacitance vessel volume. The present study confirmed previous altitude studies demonstrating that MFV increased in the MCA, 7,11,19,20 increasing more in AMS. ${ }^{11,19,20}$ Previous studies also revealed that cerebral MFV increased with moderate exercise at altitude but decreased with intense exercise in subjects developing $\mathrm{AMS},{ }^{20,21}$ indicating diminished metabolic reserve or hypoxemic tolerance. In animals, hypoxic drive was demonstrated to have nonlinear relationships to increased $\mathrm{CBF}^{22}$ regardless of hypocarbia from hyperventilation. ${ }^{23}$ Previous altitude studies dem- onstrated decreased carbon dioxide response or capacitance vessel reserve $\mathrm{e}^{20,24}$ in all subjects, including native Sherpas, regardless of AMS symptoms. ${ }^{24}$ A clear relationship exists at altitude between MFV and capacitance vessel reserve.

Capacitance vessel dilation from hypoxemic stress will improve minute oxygen delivery ${ }^{24}$ by increasing overall CBF. Capacitance vessel reserve is mediated by myogenic contraction not affected by hypoxia but cannot override the potent effects of hypoxic vasodilation itself. $^{25}$ Vasodilation is affected by metabolic alterations caused by hypoxemia, including increased cerebrospinal fluid $\mathrm{pH},{ }^{26}$ increased hematocrit, ${ }^{27}$ increased hemoglo-

Table 2. Transcranial Doppler, dynamic vascular analysis, and vascular endothelial growth factor (VEGF) changes noted from sea level to altitude $(4300 \mathrm{~m})$ between the symptomatic $(\mathrm{Sx})$ and asymptomatic (Asx) groups $\dagger$

\begin{tabular}{|c|c|c|c|c|c|c|c|c|}
\hline \multirow[b]{2}{*}{ Changes } & \multicolumn{4}{|c|}{ Sx group } & \multicolumn{4}{|c|}{ Asx group } \\
\hline & $n$ & Mean & $S D$ & $\%$ change & $n$ & Mean & $S D$ & $\%$ change \\
\hline MFV sea level & 3 & 44.61 & 9.12 & & 6 & 46.67 & 3.84 & \\
\hline MFV altitude & 3 & 62.4 & 12.59 & +39.9 & 6 & 57.27 & 4.1 & +22.7 \\
\hline ln SA sea level & 3 & 6.14 & 0.27 & & 6 & 6.04 & 0.31 & \\
\hline ln SA altitude & 3 & 6.27 & 0.2 & +2.1 & 6 & 6.44 & 0.14 & +6.6 \\
\hline Pl sea level & 3 & 0.94 & 0.07 & & 6 & 0.9 & 0.06 & \\
\hline Pl altitude & 3 & 0.79 & 0.12 & +15.3 & 6 & 1 & 0.12 & +10.9 \\
\hline VEGF sea level & 2 & 0.06 & 0.03 & & 6 & 0.07 & 0.04 & \\
\hline VEGF altitude & 2 & 0.08 & 0 & +29.6 & 6 & 0.11 & 0.04 & +62.6 \\
\hline DCI sea level & 3 & 0.14 & 0.02 & & 6 & 0.13 & 0.01 & \\
\hline DCI altitude & 3 & 0.1 & 0.02 & +28.6 & 6 & 0.11 & 0.01 & -15.4 \\
\hline DFI sea level* & 3 & 47.72 & 10.21 & & 6 & 52.06 & 5.24 & \\
\hline DFI altitude* & 3 & 81.22 & 27.9 & +70.2 & 6 & 58.19 & 8.48 & +11.8 \\
\hline DPI sea level & 3 & 6.57 & 0.45 & & 6 & 6.73 & 0.42 & \\
\hline DPI altitude & 3 & 8.03 & 1.34 & +22.2 & 6 & 6.54 & 0.8 & -2.8 \\
\hline
\end{tabular}

$* P<.05$.

$\dagger$ MFV indicates mean flow velocity; SA, systolic upstroke acceleration; PI, index of pulsatility; DCI, dynamic compliance index; DFI, dynamic flow index; and DPI, dynamic pressure index. 


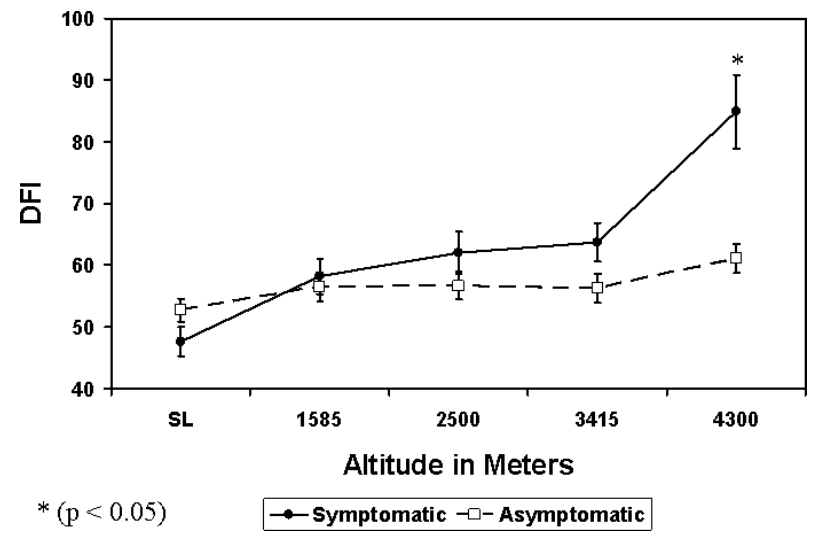

Figure 4. Effect of altitude on dynamic flow index (DFI). The DFI (mean flow velocity/index of pulsatility) significantly increased in the symptomatic group compared with the asymptomatic group $\left({ }^{*} P<.05\right.$; paired-samples $t$ test).

bin, and decreased nitric oxide, ${ }^{28}$ partially explaining acclimatization and stabilization of $\mathrm{CBF}$ at altitude. One possible mechanism for the development of AMS is impairment of endothelial gap junction function secondary to hypoxemia ${ }^{3,29-32}$ characterized by increased permeability to macromolecules and increasing interstitial free water, which may result in high-altitude cerebral ede$\mathrm{ma}^{33}$ and high-altitude pulmonary edema as well as expansion of capacitance vessel volume.

High-altitude cerebral edema may be secondary to increased endothelial permeability of cerebral capacitance or small vessels. ${ }^{12,33}$ Hypoxemic stress increases production of VEGF $33,34,35$ and VEGF receptors, ${ }^{5}$ which increase vascular permeability. Levels of VEGF correlate with altitude symptoms compared with sea level controls; however, at altitude, VEGF did not correlate with these same symptoms. ${ }^{5}$ This suggests variable responses to increasing VEGF, as suggested by our cohort. Our increase in VEGF did not reach significance but suggested an oxidative stress. Vascular endothelial growth factor increases vessel permeability. ${ }^{33}$ Increased dysfunction of the endothelial tight junctions and adherens junctions with increasing oxidative stress is known. A recent study involving rats showed direct evidence of increased permeability to larger molecular weight compounds with hypoxia. ${ }^{36}$ The increased fenestrations in the endothelial barrier leak serum proteins into interstitial spaces, pulling free water. This may explain the drop in serum VEGF levels observed in our Sx subjects. If these "vascular holes" open, then a drop in intravascular pressure would occur in capacitance vessels, thereby diminishing capillary perfusion pressure and conductance vessel resistance as observed in this study. As resistance drops, flow increases to maintain adequate minute perfusion of the capillaries. Interestingly, if enough of these

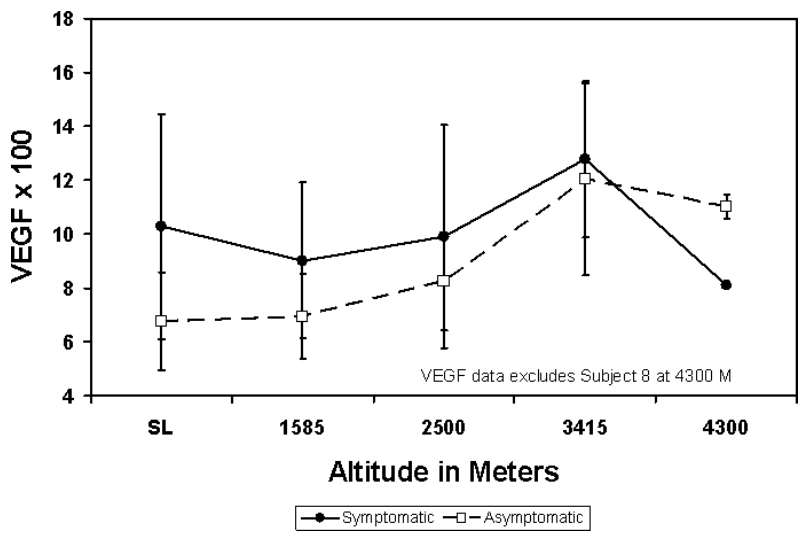

Figure 5. Effect of altitude on serum vascular endothelial growth factor (VEGF). Serum VEGF levels dropped initially at $1585 \mathrm{~m}$ in the symptomatic (Sx) group, then the levels for both the Sx group and the asymptomatic (Asx) group increased until $3415 \mathrm{~m}$, where the Sx group fell precipitously and the Asx group had a slight decline. These findings were not significant.

gaps were open, a relative drop in intraluminal pressure of a "closed" vascular system would occur because this new state would represent an "open" system, causing increased flow with less work provided by the conductance system to maintain the pressure gradient. This correlates with our findings that the PI in conductance vessels diminished, as did the vessel tone (decreased DCI) secondary to increased vessel volume (increased DFI) in the Sx group. The appropriate compensatory mechanism would increase capacitance vessel myogenic tone to increase intravascular pressure of this now-open system and increase forward perfusion pressure, thereby increasing the pressure gradient across the capillary beds. This compensatory change was evident in the Asx group but not in the Sx group.

Our study differs from other TCD-derived velocimetry studies at altitude in that holocephalic (including the posterior circulation) changes were monitored in contrast to other studies of an isolated cerebral vessel segment. This approach demonstrated that changes in CBF at altitude are holocephalic and that changes in the posterior circulation are present. Cerebral blood flow increases as a result of a medullary response to hypoxia ${ }^{37}$ mediated by a relay through the thalamus to dilate cortical vessels. This underscores the importance of monitoring posterior circulation flow when studying the pathophysiology of AMS.

In this cohort, changes in the conductance vessel SA and DCI, as markers of perfusion pressure and vessel tone, respectively, correlated with symptom scores and serum VEGF levels. Less symptomatic subjects had higher VEGF scores, with the ability to maintain cere- 
brovascular compliance (ie, higher DCI and perfusion pressure, SA). Symptomatic subjects had increased vascular tone with lower VEGF levels, suggesting increased vascular tone reflected as decreased DCI or PI. The most striking finding in our study was the reverse relationship of symptom scores to the PI. As PI diminished (lower vascular resistance secondary to expansion of capacitance vessel volume), symptoms increased.

This study was underpowered to show significant group changes, a possible type 2 error. Although serum VEGF levels were trending, this study suggests that studies with more subjects would show significant differences in VEGF levels between the symptom groups. We run the risk of a type 1 error given the number of subjects in this study. Pooling of all vessel data minimized this risk.

In summary, this DVA-enabled study indicates that acute mountain sickness is associated with altered cerebrovascular kinetics and volume expansion as a function of dysfunctional capacitance vessels. At altitude, increased permeability of the vascular endothelium from opening of endothelial junctions in capacitance vessels most likely precedes increasing symptoms and eventually high-altitude cerebral edema and high-altitude pulmonary edema. Dynamic vascular analysis proved to be a sensitive tool for measuring and elucidating the vascular physiology behind changes in CBF dynamics associated with symptomatic altitude illness. Potential therapies directed at maintaining the integrity of endothelial junctions and capacitance vessel tone to prevent acute altitude sickness or other hypoxemic states could be noninvasively monitored with DVA.

\section{Acknowledgments}

The authors thank Alan Faden, MD, for his review and comments regarding the manuscript and James Zimble, MD, Barry Wolcott, MD, and Fred Cecere, MD, for their support. Dynamic vascular analysis is a processing method for Doppler data provided by New Health Sciences Inc. Dynamic vascular analysis, dynamic flow index, dynamic pressure index, and dynamic compliance index are trademarks of New Health Sciences Inc, Rockville, MD 20850, (240)453-9191.

\section{References}

1. West JB. Oxygen enrichment of room air to improve wellbeing and productivity at high altitude. Int J Occup Environ Health. 1999;5:187-193.

2. Burtscher M. High altitude headache: epidemiology, pathophysiology, therapy and prophylaxis. Wien Klin Wochenschr. 1999;111:830-836.
3. Schoch HJ, Fischer S, Marti HH. Hypoxia-induced vascular endothelial growth factor expression causes vascular leakage in the brain. Brain. 2002;125(pt 11):2549-2557.

4. Appenzeller O, Minko T, Pozharov V, et al. Gene expression in the Andes; relevance to neurology at sea level. $J$ Neurol Sci. 2003;207:37-41.

5. Maloney J, Wang D, Duncan T, Voelkel N, Ruoss S. Plasma vascular endothelial growth factor in acute mountain sickness. Chest. 2000;118:47-52.

6. Mentzer RM, Rubio R, Berne RM. Release of adenosine by hypoxic canine tissue and its possible role in pulmonary circulation. Am J Physiol. 1975;229:1625-1631.

7. Jensen JB, Wright AD, Lassen NA, et al. Cerebral blood flow in acute mountain sickness. J Appl Physiol. 1990;69: 430-433.

8. Yang YB, Sun B, Yang Z, Wang J, Pong Y. Effects of acute hypoxia on intracranial dynamics in unanesthetized goats. J Appl Physiol. 1993;74:2067-2071.

9. Berre J, Vachiery JL, Moraine JJ, Naeije R. Cerebral blood flow velocity responses to hypoxia in subjects who are susceptible to high-altitude pulmonary oedema. Eur J Appl Physiol. 1999;80:260-263.

10. Ter MA, Beydon L, Ursino M, Gardette B, Gortan C, Richalet JP. Doppler study of middle cerebral artery blood flow velocity and cerebral autoregulation during a simulated ascent of Mount Everest. Wilderness Environ Med. 2001;12:175-183.

11. Otis SM, Rossman ME, Schneider PA, Rush MP, Ringelstein EB. Relationship of cerebral blood flow regulation to acute mountain sickness. J Ultrasound Med. 1989;8: 143-148.

12. Hackett PH. The cerebral etiology of high-altitude cerebral edema and acute mountain sickness. Wilderness Environ Med. 1999;10:97-109.

13. Crutchfield KE, Razumovsky AY, Tegeler CH, Mozayeni BR. Differentiating vascular pathophysiological states by objective analysis of flow dynamics. J Neuroimaging. 2004;14:97-107.

14. Speilberger CD, Gorsuch RL. Manual for the State-Trait Anxiety Inventory (STAI). Palo Alto, Calif: Consulting Psychologists Press; 1970.

15. Hardt J, Gerbershagen HU, Franke P. The Symptom Checklist SCL-90-R: its use and characteristics in chronic pain patients. Eur J Pain. 2002;4:137-148.

16. Savourey G, Guinet A, Besnard Y, Garcia N, Hanniquet A, Bittel J. Are the laboratory and field conditions observations of acute mountain sickness related? Aviat Space Environ Med. 1997;68:895-899.

17. Kao WF, Kuo CC, Hsu TF, et al. Acute mountain sickness in Jade Mountain climbers of Taiwan. Aviat Space Environ Med. 2002;73:359-362.

18. Aaslid R, Markwalder TM, Nornes H. Noninvasive transcranial Doppler ultrasound recording of flow velocity in basal cerebral arteries. J Neurosurg. 1982;57:769-774.

19. Baumgartner RW, Bartsch P, Maggiorini M, Waber U, Oelz O. Enhanced cerebral blood flow in acute mountain sickness. Aviat Space Environ Med. 1994;65:726-729. 
20. Jansen GF, Krins A, Basnyat B. Cerebral vasomotor reactivity at high altitude in humans. J Appl Physiol. 2003; 86:681-686.

21. Huang SY, Sun S, Droma T, et al. Internal carotid arterial flow velocity during exercise in Tibetan and Han residents of Lhasa. J Appl Physiol. 2003;73:2638-2642.

22. Jensen JB, Sperling B, Severinghaus JW, Lassen NA. Augmented hypoxic cerebral vasodilation in men during 5 days at 3,810 m altitude. J Appl Physiol. 1996;80:12141218.

23. Lassen NA. Increase of cerebral blood flow at high altitude: its possible relation to AMS. Int J Sports Med. 1992; 13(suppl 1):S47-S48.

24. Jansen GF, Krins A, Basnyat B, Bosch A, Odoom JA. Cerebral autoregulation in subjects adapted and not adapted to high altitude. Stroke. 2000;31:2314-2318.

25. Lui Y, Harder DR, Lombard JH. Interaction of myogenic mechanisms and hypoxic dilation in rat middle cerebral arteries. Am J Physiol Heart Circ Physiol. 2002;283: H2276-H2281.

26. Weiskopf RB, Gabel RA, Fencl V. Alkaline shift in lumbar and intracranial CSF in man after 5 days at high altitude. J Appl Physiol. 1976;41:93-97.

27. Clench J, Ferrell RE, Schull WJ. Effect of chronic altitude hypoxia on hematologic and glycolytic parameters. Am J Physiol. 1982;242:R447-R451.

28. Schechter AN, Gladwin MT. Hemaglobin and the paracrine and endocrine functions of nitric oxide. $N$ Engl $J$ Med. 2003;348:1483-1485.
29. Abbott NJ. Astrocyte-endothelial interactions and bloodbrain barrier permeability. J Anat. 2002;200:629-638.

30. Brown RC, Davis TP. Calcium modulation of adherens and tight junction function. Stroke. 2002;33:1706-1711.

31. Pachter JS, De Vries HE, Fabry Z. The blood-brain barrier and its role in immune privilege in the central nervous system. J Neuropathol Exp Neurol. 2003;62:593-604.

32. Van Hinsbergh VW, Van Nieuw Amerongen GP. Intracellular signalling involved in modulating human endothelial barrier function. J Anat. 2002;200:549-560.

33. Severinghaus JW. Hypothetical roles of angiogenesis, osmotic swelling, and ischemia in high-altitude cerebral edema. J Appl Physiol. 1995;79:375-379.

34. Walter R, Maggiorini M, Scherrer U, Contesse J, Reinhart WH. Effects of high-altitude exposure on vascular endothelial growth factor levels in man. Eur J Appl Physiol. 2001;85:113-117.

35. Maloney J, Wang D, Duncan T, Voelkel N, Ruoss S. Plasma vascular endothelial growth factor in acute mountain sickness. Chest. 2000;118:47-52.

36. Witt K, Marks KS, Hom S, Davis TP. Effects of hypoxiareoxygenation on rat blood-barrier permeability and tight junction protein expression. Am J Physical Heart Circ Physiol. 2003 Dec;285(6):H2820-31.

37. Golanov EV, Christensen JR, Reis DJ. Neurons of a limited subthalamic area mediate elevations in cortical cerebral blood flow evoked by hypoxia and excitation of neurons of the rostral ventrolateral medulla. J Neurosci. 2001; 21:4032-4041. 